\title{
Design of Equivalent Circuits and Characterization Strategy for $n$-Input Coupled Inductors
}

\author{
Xavier Margueron and Jean-Pierre Keradec, Member, IEEE
}

\begin{abstract}
This paper begins with the definition of ideal coupler, the justification of negative inductance concept and the establishment of various equivalences between circuits. Then, an energy-based recursive method for designing for the equivalent circuit of an $n$-input magnetic system is presented and the leakage transformer concept is introduced. The obtained circuits allow easy checking of inequalities that inductance matrix elements must satisfy. Advantage is then taken from winding identity and dominant coupling to simplify circuit design. This new knowledge is applied to design equivalent circuits for a three-phase inductor and a three-column six-winding transformer. Finally, key points of a strategy intended to experimental identification of all circuit elements are given.
\end{abstract}

Index Terms-Equivalent circuit, identification, magnetic coupling, transformer.

\section{INTRODUCTION}

$\mathbf{T}$ ODAY, power electronic design relies on electronic simulation to avoid, or at least to reduce, prototyping. To reach this goal, accurate models of every component are needed. From these components, transformers are among the most complex, especially when they comprise four windings or more. This paper focuses on the transformer and general methods are presented to design its equivalent circuit. Despite the fact that all parameters are assumed to depend neither on currents nor on frequency, a large part of this paper remains applicable in practical situations.

A transformer generally has good power efficiency, thus its equivalent circuit is mainly reactive. Moreover, its coupling is magnetic on the low-frequency side and electrostatic in high frequency. Hence, it is assumed that its equivalent circuit can be split into two parts: a magnetic part and an electrostatic one wired in parallel at all external terminals only. Of course, resistors must be added to this elementary circuit to account for all kind of losses. To sum up, one understands why representation of magnetic coupling supplies the backbone of the equivalent

Paper IPCSD-06-084, presented at the 2004 Industry Applications Society Annual Meeting, Seattle, WA, October 3-7, and approved for publication in the IEEE TRANSACTIONS ON INDUSTRY APPLICATIONS by the Power Electronics Devices and Components Committee of the IEEE Industry Applications Society. Manuscript submitted for review September 26, 2005 and released for publication September 25, 2006. This work was supported by the Thales Company.

The authors are with the Laboratoire d'Electrotechnique de Grenoble, Institut National Polytechnique de Grenoble (INPG)/University Joseph Fourier (UJF) Unité Mixte de Recherche (UMR)-Centre National de la Recherche Scientifique (CNRS) 5529, ENSIEG, 38402 Saint-Martin-d'Hères Cedex, France (e-mail: Jean-Pierre.Keradec@leg.ensieg.inpg.fr).

Color versions of one or more of the figures in this paper are available online at http://ieeexplore.iee.org.

Digital Object Identifier 10.1109/TIA.2006.887001 circuit of the transformer: At low and medium frequencies, a transformer's electrical behavior is mainly due to its magnetic coupling.

Despite the fact that equivalent circuits are available in the literature to represent magnetic coupling of two- and threewinding transformers, no general method of design has yet been published for more windings [1]. Some years ago we published such a method in French [2] which was only partially reported in English [3]. Our goal consists of representing the inductance matrix by an equivalent circuit. From a physical point of view, energy considerations are of central importance in our developments. The topology of the resulting circuit depends only on inductance matrix size and links (exact or approximated) between elements. In this approach, the wellknown ideal transformer, or "coupler," plays a new role: in association, it allows a base change to be made on voltages or currents. Components included in the final circuit can be deduced either from measurement with no need for additional information (regarding: size, technology, etc.) or from electromagnetic simulation or even, sometimes, through analytical computation.

In the past, we used the extended cantilever model [2], [4]. It is general, but the resulting topology is not easily clarified when the component owns simple properties. The method introduced here permits us to take advantage of such properties to simplify the design of the equivalent circuit. Two common properties are investigated. First, identity of two windings leads to simplifications whether or not they are wired together. Second, when a winding is coupled with another stronger than with all others (dominant coupling), simplifications can also take place. In both cases, methods are given to consequently simplify the equivalent circuit.

Practical examples are presented including a three-phase inductor and a three-column $2 \times 3$-winding 12.5-kVA transformer. An investigation regarding the best choice of measurements aimed at accurately identifying all components included in the equivalent circuit is included.

\section{COMmon Equivalent Circuits}

\section{A. Simple and Multiple Couplers}

In order to represent a transformer, a "two-winding ideal transformer" or "coupler" must be introduced. The chosen picture (Fig. 1) avoids allusion to a winding so it can also be used for electrostatic coupling. The coupling ratio $\eta$ is defined by

$$
V_{1}=\eta_{21} V_{2} \quad \text { and } \quad I_{2}+\eta_{21} I_{1}=0
$$




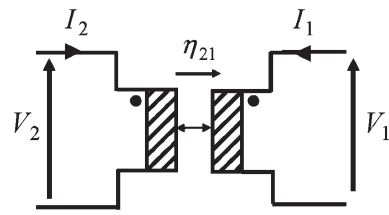

Fig. 1. Coupler.

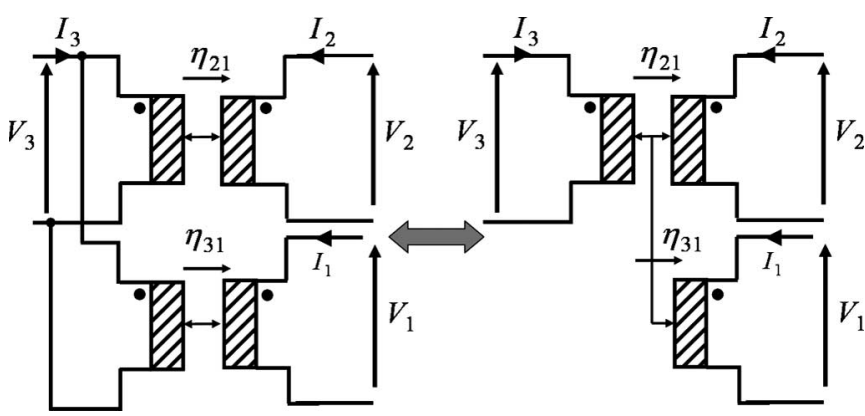

Fig. 2. Multiple-port coupler.

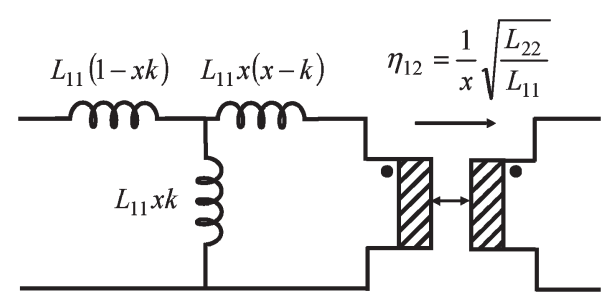

Fig. 3. Equivalent circuit of a two-winding transformer.

The black dot at each coupler input indicates positive voltage orientation. The sign of the coupling ratio can be changed by moving the dot to the opposite terminal of the same input. For each input, positive orientation of the current is indicated by an arrow. It enters the coupler by the black point side.

For multiple winding transformers, assemblies of simple couplers are conveniently replaced by a single multiple-port coupler (Fig. 2). The circuit shown is equivalent to a pair of simple couplers having their primary sides wired in parallel. $V_{1}=\eta_{31} V_{3}, V_{2}=\eta_{32} V_{3}$, and $I_{3}+\eta_{31} I_{1}+\eta_{32} I_{2}=0$.

\section{B. Common Equivalent Circuit of a Two-Winding Transformer}

In the common equivalent circuit (Fig. 3), black dots are placed so the mutual inductance $L_{12}$ is positive. Values are given in terms of matrix elements but $L_{12}$ is expressed as a function of coupling coefficient $k$ according to

$$
L_{12}=k \sqrt{L_{11} L_{22}} .
$$

Because an inductance matrix is always symmetrical, the matrix of a two-winding transformer has three independent elements. Despite this, in Fig. 3 four values appear so one of them can be chosen freely. For this reason, we have introduced an $x$ factor that allows coupling ratio tuning. The inductance matrix attached to the circuit in Fig. 3 does not depend on the $x$ value.

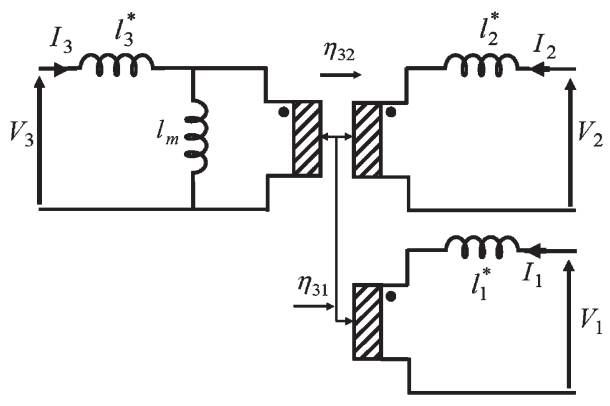

Fig. 4. Commonly used equivalent circuit for a three-winding transformer.

Serial (leakage) inductances depend on the $x$ factor. According to $x=k, 1$ or $1 / k$, leakages appear on the primary side, split into two equal parts or on the secondary side. Indeed, these equivalences recall that, generally, we only know that leakages are between windings. Sharing them between the two windings needs extra information regarding field shape. Moreover, if $x$ is taken outside the aforementioned narrow interval, one leakage inductance is negative despite all the measurable inductances remaining positive. Although surprising, there is no physical reason to reject such a value which often appears when the coupling ratio is taken equal to the turns ratio.

Looking at the same circuit when $x=k$, it is easy to derive that the coupling coefficient can be deduced from open- $\left(L_{\mathrm{o}}\right)$ and short- $\left(L_{\mathrm{sc}}\right)$ circuit inductances seen from the primary winding according to (2).

The same expression is valid for measurements acquired from the other winding

$$
k=\sqrt{1-\frac{L_{\mathrm{sc}}}{L_{\mathrm{o}}}} .
$$

Comparing circuits obtained for $x=k$ and $x=1 / k$ leads to a convenient equivalence which allows the leakage inductance to move from downstream to upstream position (and inversely) relative to magnetizing inductance.

To conclude, it should be underlined that the turns ratio is sometimes neither known nor measurable. Even in such a case, matrix elements are always measurable. Fig. 3 gives convenient circuits, provided $x$ is chosen among the three special values introduced above.

\section{Common Equivalent Circuit of a Three-Winding Transformer}

To represent a three-winding transformer, numerous authors [5] use the equivalent circuit given in Fig. 4. While its inductance matrix has six independent coefficients, this circuit is characterized by six parameters that, consequently, are fully determined.

To express the six parameters in terms of inductance matrix elements, one can set the magnetic energy (3) stored in the circuit equal to that stored in the transformer [6].

$$
W=\frac{1}{2} \sum_{i, j=1}^{3} L_{i j} I_{i} I_{j} \quad \text { with } \quad L_{i j}=L_{j i}
$$



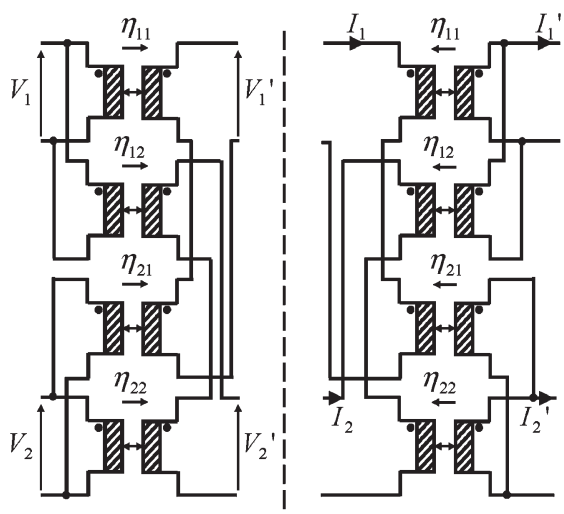

Fig. 5. Base change examples for voltages (left) and currents (right).

and

$$
\begin{aligned}
2 W & =l_{1}^{*} I_{1}^{2}+l_{2}^{*} I_{2}^{2}+l_{3}^{*} I_{3}^{2}+l_{m}\left(\eta_{13} I_{1}+\eta_{23} I_{2}+I_{3}\right)^{2} \\
l_{1}^{*} & =L_{11}-\frac{L_{12} L_{13}}{L_{23}} \quad l_{2}^{*}=L_{22}-\frac{L_{12} L_{23}}{L_{13}} \\
l_{3}^{*} & =L_{33}-\frac{L_{13} L_{23}}{L_{12}} \quad l_{m}=\frac{L_{13} L_{23}}{L_{12}} \\
\eta_{13} & =\frac{L_{12}}{L_{23}} \quad \eta_{23}=\frac{L_{12}}{L_{13}} .
\end{aligned}
$$

As for two windings, some inductances may be negative. Unfortunately, there is no adjustable parameter and, consequently, there is no chance of avoiding negative values. Therefore, despite the fact that this equivalent circuit is physically correct, it may be difficult to introduce it in some simulation software.

This kind of representation, which associates couplers with one magnetizing inductance and one leakage inductance for each winding, becomes unsuitable for four windings and more because of its insufficient number of adjustable parameters. For example, a four-winding transformer has a ten independent coefficient inductance matrix but related equivalent circuit only introduces eight adjustable parameters.

\section{RECURSIVE APPROACH}

\section{A. Base Change for Currents and Voltages}

It is known that adequate base change greatly simplifies matrix calculation. For two windings, the respective relations are

$$
\begin{aligned}
& V_{1}^{\prime}=\eta_{11} V_{1}+\eta_{21} V_{2} \\
& V_{2}^{\prime}=\eta_{12} V_{1}+\eta_{22} V_{2}
\end{aligned}
$$

and

$$
\begin{aligned}
& I_{1}^{\prime}=\eta_{11} I_{1}+\eta_{12} I_{2} \\
& I_{2}^{\prime}=\eta_{21} I_{1}+\eta_{22} I_{2}
\end{aligned}
$$

Fig. 5 shows a circuit representation of this base change using couplers. Obviously, this technique can be extended to any number of currents or voltages.

\section{B. Magnetostatic Energy and Quadratic Functions}

As long as an $n$-input transformer behaves linearly, its magnetic energy is a quadratic function of input currents. This dependence (3) is often expressed in matrix form (4). In this expression, $[I]$ is a column matrix that gathers up all input currents $I_{i}$ and exponent $t$ indicates the matrix transposition

$$
W=\frac{1}{2}[I]^{t}[L][I] .
$$

Matrix $[L]$ is real and symmetrical. As a consequence, owing to a correct base change, it can appear as diagonal. In this new base, $[L]$ and $[I]$ become $\left[L^{\prime}\right]$ and $\left[I^{\prime}\right]$ and expression (4) is modified to become (5)

$$
W=\frac{1}{2} \sum_{i=1}^{n} L_{i i}^{\prime} I_{i}^{\prime 2} .
$$

Stored energy looks like a set of inductances $L_{i i}^{\prime}$ gone through by currents $I^{\prime}$. Since the energy of the system is either positive or zero whatever the currents $I_{i}$, this also holds whatever the currents $I_{i}^{\prime}$; so inductances $L_{i i}^{\prime}$ are all positive. Moreover, energy (4) being positive or null whatever be $[I]$, matrix $[L]$ is definite and positive so every principal sub-matrix has a positive determinant.

\section{Schmidt Orthogonalization and General Circuit Diagram}

As noted above, the magnetic energy expression looks simpler if the current base is well chosen. Moreover, Section III-A teaches us how to undertake a base change with $n^{2}$ couplers. Although we are ready to design an equivalent circuit based on these considerations, we will make use of Schmidt's orthogonalization method [7] to reduce the coupler number. According to this method, if $n$ currents $I_{i}$ are independent, it is possible to find $n(n-1) / 2$ coefficients $\lambda_{i j}$ to obtain currents $I_{i}^{\prime}$ that make the matrix $\left[L^{\prime}\right]$ diagonal. These currents are written as follows:

$$
\begin{aligned}
& I_{1}^{\prime}=I_{1} \\
& I_{2}^{\prime}=\lambda_{12} I_{1}^{\prime}+I_{2} \\
& I_{3}^{\prime}=\lambda_{13} I_{1}^{\prime}+\lambda_{23} I_{2}^{\prime}+I_{3}
\end{aligned}
$$

According to this method, Fig. 6 shows how to build an equivalent circuit in which each current $I^{\prime}$ flows through a positive inductance. The process is recursive: in the $n$-winding transformer, the dotted line framework surrounds an $n-1$ winding transformer.

\section{General Properties of These Circuits}

These circuits are defined by $n(n+1) / 2$ parameters that can be split into $n(n-1) / 2$ coupling ratios and $n$ positive inductances. In this way, the parameter number is equal to the number of independent elements of the inductance matrix. The associated inductance matrices are also established by recursion 


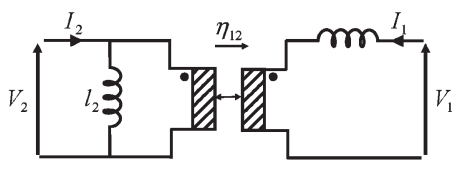

2 Windings

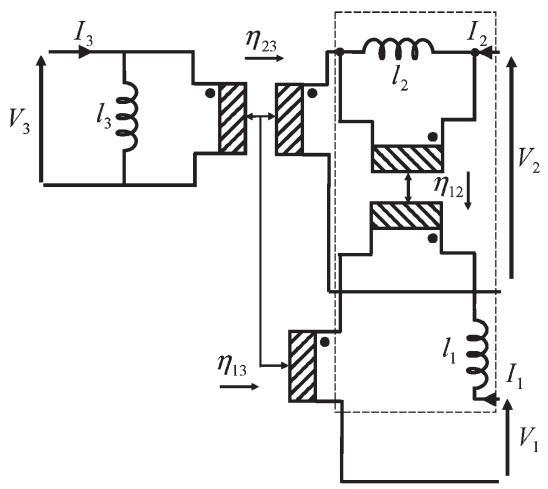

3 Windings

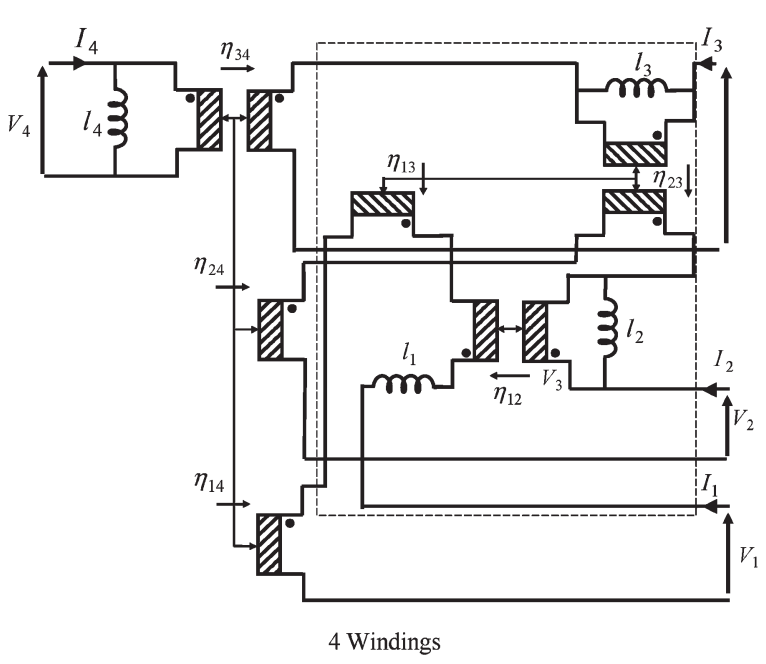

Fig. 6. Equivalent circuits for $n$-winding transformers.

one winding

$$
\left[L_{1}\right]=l_{1} \quad 1 \text { element matrix }
$$

two windings

$$
\left[L_{2}\right]=\left[\begin{array}{cc}
{\left[L_{1}\right]} & 0 \\
0 & 0
\end{array}\right]+l_{2}\left[\begin{array}{cc}
\eta_{12}^{2} & \eta_{12} \\
\eta_{12} & 1
\end{array}\right]
$$

and

three windings

$$
\left[L_{3}\right]=\left[\begin{array}{ccc}
{\left[L_{2}\right]} & & 0 \\
& & 0 \\
0 & 0 & 0
\end{array}\right]+l_{3}\left[\begin{array}{ccc}
\eta_{13}^{2} & \eta_{13} \eta_{23} & \eta_{13} \\
\eta_{13} \eta_{23} & \eta_{23}^{2} & \eta_{23} \\
\eta_{13} & \eta_{23} & 1
\end{array}\right] .
$$

Currents flowing in the inductances of the four-winding circuit are expressed as follows. Resemblance to the above Schmidt's relations is evident

$$
\begin{aligned}
& I_{l_{1}}=I_{1} \\
& I_{l_{2}}=\eta_{12} I_{1}+I_{2} \\
& I_{l_{3}}=\eta_{13} I_{1}+\eta_{23} I_{2}+I_{3} \\
& I_{l_{4}}=\eta_{14} I_{1}+\eta_{24} I_{2}+\eta_{34} I_{3}+I_{4} .
\end{aligned}
$$

In all these circuits, the left side inductance can be described as the magnetizing inductance while the transformer in the framework is denoted the "leakage transformer." It must be noted that leakage inductances are generally coupled. However, because most of the coupling between two windings is accounted for by the left couplers, couplings inside the leakage transformer are often weak and they are sometimes negligible.

Magnetic energy $W$ stored in an $n$-winding transformer is now written as in (6)

$$
W=\frac{1}{2} \sum_{i=1}^{N} l_{i}\left(\sum_{j=1}^{i-1} \eta_{i j} I_{j}+I_{i}\right)^{2} .
$$

\section{E. Parameter Identification}

If a four-winding transformer (Fig. 6) is taken as an example, it is easy to see how each of its parameters can be directly measured or deduced from an electromagnetic simulation:

$$
\begin{gathered}
l_{1}=\begin{array}{c}
\text { inductance seen from } 1 \text { with all other inputs } \\
\text { short circuited; }
\end{array} \\
l_{2}=\text { inductance seen from } 2 \text { with all other inputs } \\
\text { short circuited except } 1 ; \\
l_{3}=\text { inductance seen from } 3 \text { with all other inputs } \\
\text { short circuited except } 1 \text { and } 2 ; \\
l_{4}=\text { inductance seen from } 4 \text { with all other inputs } \\
\text { short circuited except } 1,2 \text {, and } 3 .
\end{gathered}
$$

These inductances are directly measurable so they are positive. Coupling ratios are also directly measurable:

$$
\begin{gathered}
\eta_{i 4}=\frac{V_{i}}{V_{4}} \text { supplying } 4, \text { other inputs open circuited; } \\
\eta_{i 3}=\frac{V_{i}}{V_{3}} \text { supplying } 3, \text { other inputs open circuited } \\
\text { except } 4 \text { short circuited; }
\end{gathered}
$$

$\eta_{i 2}=\frac{V_{i}}{V_{2}}$ supplying 2 , other inputs open circuited

except 4 and 3 short circuited.

The determination of the ten parameters in terms of the ten independent coefficients of the inductance matrix is accomplished using identification between (3) and (6). The calculation is simpler if identification is taken back: $L_{44}, L_{43}, L_{42}$, $L_{41}, L_{33} \ldots$..

\section{SimplificATIONS}

\section{A. Identity of Two Windings}

It is now supposed that, among transformer windings, two are identical: they can be exchanged without changing component behavior. To study the consequences of this property let us assume windings 1 and 2 of a four-winding transformer are 


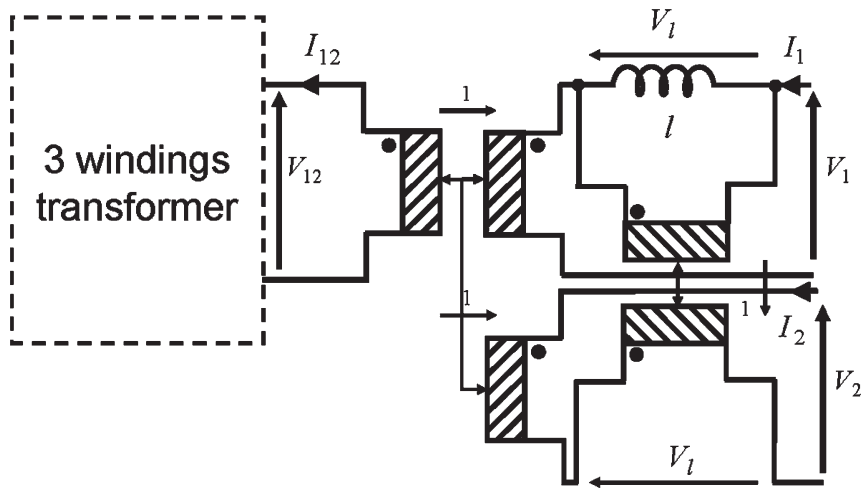

Fig. 7. Equivalent circuit with two identical insulated windings.

identical. In this case, permutation between currents $I_{1}$ and $I_{2}$ must not affect the stored energy $W$ whatever the values of $I_{3}$ and $I_{4}$. Comparing (3) before and after the exchange, it appears that three equalities must be satisfied

$$
L_{11}=L_{22} \quad L_{13}=L_{23} \quad L_{14}=L_{24} .
$$

The first consequence of this identity is that inductance matrix has seven independent elements instead of ten so an equivalent circuit, characterized by seven parameters only, can be designed. $W$ is examined after $L_{11}, L_{13}$, and $L_{14}$ have been removed using the previous equalities

$$
\begin{aligned}
W=\frac{1}{2} L_{22}( & \left.I_{1}^{2}+I_{2}^{2}\right)+\frac{1}{2} L_{33} I_{3}^{2}+\frac{1}{2} L_{44} I_{4}^{2} \\
& +L_{12} I_{1} I_{2}+L_{23}\left(I_{1}+I_{2}\right) I_{3}+L_{24}\left(I_{1}+I_{2}\right) I_{4} .
\end{aligned}
$$

This expression is very close to that of a transformer whose three windings are supplied, respectively, by $I_{1}+I_{2}, I_{3}$, and $I_{4}$. Putting $L=\left(L_{22}+L_{12}\right) / 2$ and $l=\left(L_{22}-L_{12}\right) / 2$ it becomes

$$
\begin{aligned}
W=\frac{1}{2} L & \left(I_{1}+I_{2}\right)^{2}+\frac{1}{2} L_{33} I_{3}^{2}+\frac{1}{2} L_{44} I_{4}^{2} \\
& +L_{23}\left(I_{1}+I_{2}\right) I_{3}+L_{24}\left(I_{1}+I_{2}\right) I_{4}+\frac{1}{2} l\left(I_{1}-I_{2}\right)^{2} .
\end{aligned}
$$

Now, things look very simple: $W$ is the sum of energies stored in a three-winding transformer and in an inductance $l$ gone through by $I_{1}-I_{2}$. This property justifies the equivalent circuits shown in Figs. 7 and 8 in which all coupling ratios of the couplers shown equal 1 . Notice that, according to their expressions, $L$ and $l$ are both positive.

Both equivalent circuits need six parameters to describe the three-winding transformer and one $(l)$ to describe the external circuit. That is consistent with the number of independent elements of the inductance matrix. This method can be extended to any number of windings.

\section{B. Influence of Dominant Coupling}

Despite the fact that the previous simplification does not require any approximation, the next one does. This simplification applies when a pair of windings is strongly coupled (coupling coefficient $k$ very close to 1 ), whereas couplings of

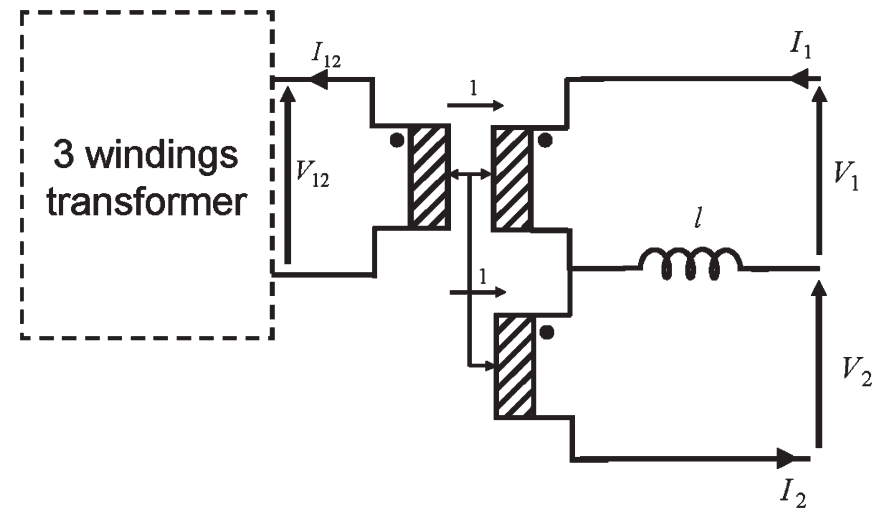

Fig. 8. Simplification for a center-tapped winding.

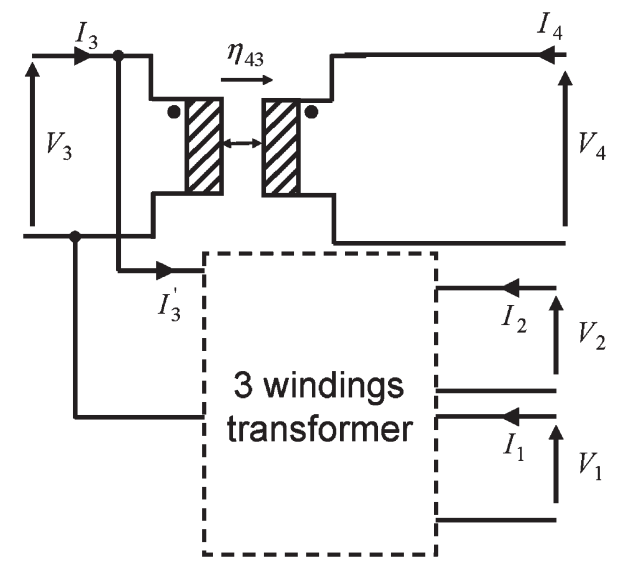

Fig. 9. Equivalent circuit with perfect coupling of two windings.

these windings with all other ones are weaker. For example, a four-winding transformer, in which windings 3 and 4 are perfectly coupled together while they are less coupled with the two others, can be represented as shown in Fig. 9. In this situation, which we call "dominant coupling," two lines (and also two columns because inductance matrix is symmetrical) are proportional; matrix (7) is singular

$$
[L]=\left[\begin{array}{cccc}
L_{11} & L_{12} & L_{13} & \eta_{43} L_{13} \\
L_{12} & L_{22} & L_{23} & \eta_{43} L_{23} \\
L_{13} & L_{23} & L_{33} & \eta_{43} L_{33} \\
\eta_{43} L_{13} & \eta_{43} L_{23} & \eta_{43} L_{33} & \eta_{43}^{2} L_{33}
\end{array}\right] .
$$

In practice, a pair of windings is never perfectly coupled. Therefore, for a more realistic approach, a leakage inductance must be introduced in series with one of the two strongly coupled windings. The exact location of this inductor does not matter, provided other windings are not too strongly coupled with the pair concerned. This inductance can even be split into two parts as shown in Fig. 14. With this extra component, the inductance matrix ends up being singular.

\section{Weak Coupling in Leakage Transformer}

A leakage transformer only adds serial impedances to the main coupling. So, according to (2), as soon as coupling 


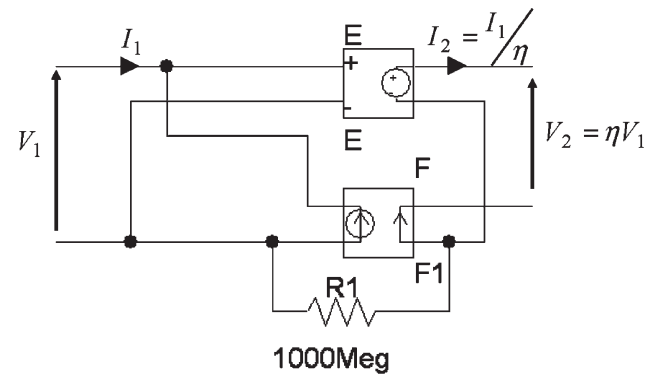

Fig. 10. Coupler representation in Pspice.

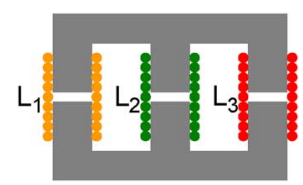

Fig. 11. Three-phase inductor.

coefficient $k<0.2$, neglecting the related coupling only introduces a $4 \%$ inaccuracy on some serial impedances.

\section{How to Use it in Circuit Simulation?}

\section{A. Coupler Representation}

Perfect couplers are the base of our representation. It can be easily represented (Fig. 10) by two controlled sources, one in voltage and the other one in current.

In order to avoid an inductive loop, it is sometimes interesting to put in series a small resistance. With this addition, a resolution algorithm manages to converge.

\section{B. Application to a Three-Phase Inductor}

When three-phase filtering is needed, two techniques are usable to insert a series inductor on each of the three wires. One can use either three separate inductors or three windings coiled on the same core. Regarding volume and weight, the latter technique performs far better so it is often chosen for high-power applications. In practice, each inductor is wound on an air-gapped core leg as shown in Fig. 11.

Obviously, this component cannot be represented accurately by three separate inductors.

Its equivalent circuit is deduced from the inductance matrix which, in this case, is very simple. Due to air gaps, magnetic flux created by one coil is quite perfectly split into two equal parts before passing through the two others coils. Noting $R$ the air gap reluctance and $N$ the turn number, matrix elements are as follows:

$$
L_{i i}=2 L \quad \text { and } \quad L_{i j}=-L \quad \text { with } \quad L=\frac{N^{2}}{3 R} .
$$

The topology of an equivalent circuit suitable for this device is obtained by merging the circuit of Fig. 7 with that of a

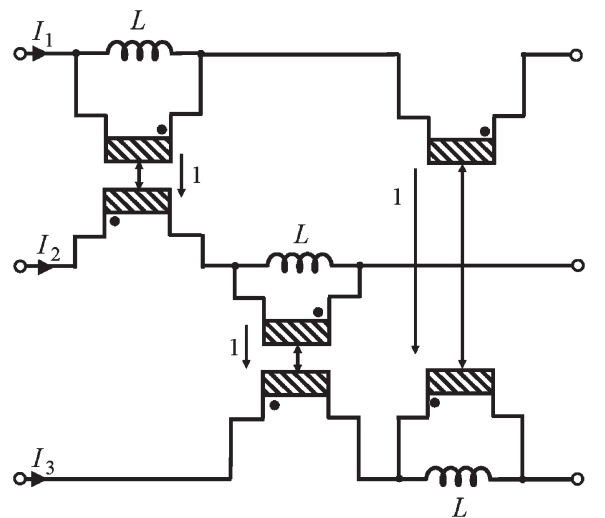

Fig. 12. Equivalent circuit for a three-phase inductor.

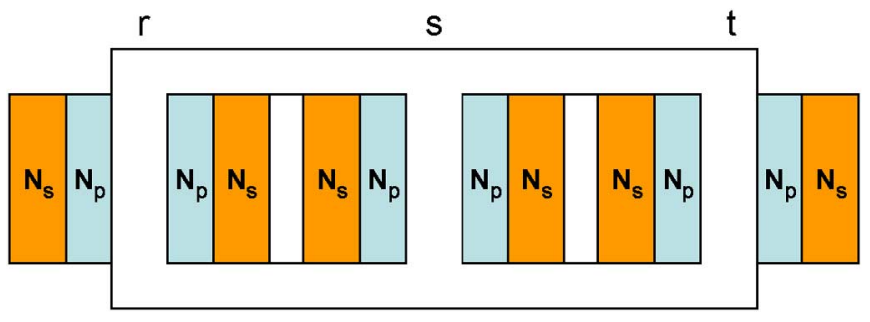

Fig. 13. Transformer geometry.

two-winding transformer (Fig. 6). Values of its components are obtained by identifying the energy $W$ stored by this circuit with the general expression (8).

Design of an equivalent circuit is not unique. For our inductor, a simpler symmetrical circuit (Fig. 12) is deduced directly from another writing (9) of $W$

$$
\begin{aligned}
& W=L\left(I_{1}^{2}+I_{2}^{2}+I_{3}^{2}\right)-L\left(I_{1} I_{2}+I_{1} I_{3}+I_{2} I_{3}\right) \\
& W=\frac{1}{2} L\left(I_{1}-I_{2}\right)^{2}+\frac{1}{2} L\left(I_{2}-I_{3}\right)^{2}+\frac{1}{2} L\left(I_{3}-I_{1}\right)^{2} .
\end{aligned}
$$

\section{Application to a $2 \times 3$-Winding Transformer}

1) Transformer Description and Measurements: The studied component is a three-column $2 \times 3$-winding 12.5-kVA transformer (Fig. 13). Its others characteristics are $V_{N 1}=83 \mathrm{~V}$ and $V_{N 2}=220 \mathrm{~V}$. Transformer columns are named $r, s$, and $t$; for example, $r_{2}$ designates secondary (winding 2) from column $r$.

2) Simplifications: At first sight, Fig. 13 suggests that columns $r$ and $t$ play the same role, and so the two windings of $r$ can be exchanged with those of $t$ with no impact on transformer behavior. It also seems highly probable that coupling between any winding with another located on the same column is stronger than with one wound on another column. Measured inductance matrix (10) clearly confirms these properties. All values are in millihenrys.

To within about $1.5 \%$, originating from measurement errors, the matrix is symmetrical, column inversion $(r$ and $t$ ) does not 


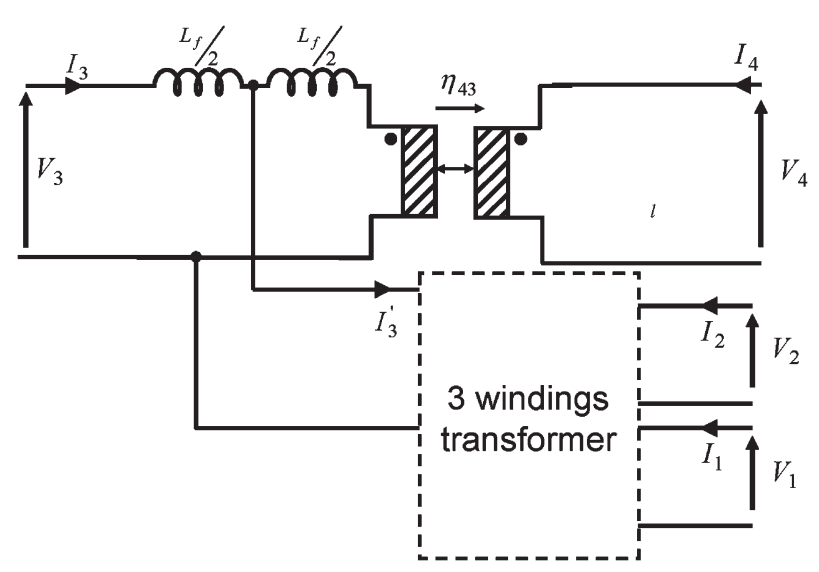

Fig. 14. Equivalent circuit with two strongly coupled windings.

affect the system, and several equalities appear between matrix coefficients

$$
\begin{aligned}
& {[L]=\begin{array}{c}
r_{1} \\
r_{1} \\
s_{1} \\
t_{1} \\
r_{2} \\
s_{2} \\
t_{2}
\end{array} \quad\left[\begin{array}{cccccc}
111 & -69 & -42 & 292 & -182 & -110 \\
-69 & 137 & -68 & -182 & 362 & -180 \\
-42 & -68 & 110 & -110 & -180 & 290 \\
292 & -182 & -110 & 775 & -482 & -292 \\
-182 & 362 & -180 & -482 & 960 & -477 \\
-110 & -180 & 290 & -292 & -477 & 770
\end{array}\right]} \\
& L_{r 1}=L_{t 1} \quad L_{r 1 s 1}=L_{t 1 s 1} \quad L_{r 1 s 2}=L_{t 1 s 2} \\
& L_{r 1 r 2}=L_{t 1 t 2} \quad L_{r 1 t 2}=L_{t 1 r 2} \quad L_{s 1 r 2}=L_{s 1 t 2} \\
& L_{s 2 r 2}=L_{s 2 t 2} \quad L_{r 2}=L_{t 2} .
\end{aligned}
$$

Values of coupling coefficients are interesting

$$
k_{r 1 r 2}=0.996 \quad k_{s 1 s 2}=0.998 \quad k_{t 1 t 2}=0.996
$$

and, with $i$ and $j=1$ or 2 .

$$
k_{\text {risj }}=-0.56 \quad k_{\text {ritj }}=-0.38 \quad k_{\text {sitj }}=-0.55 .
$$

The sign attributed to $k$ is related to mutual inductances. Hence, for two windings on different columns, mutual inductance and $k$ are negative.

Not only are the identities of columns $r$ and $t$ obvious but also dominant couplings appear for each pair of windings belonging to the same column. This gives a useful guide to finding the topology of the equivalent circuit we are looking for.

To simplify this problem, we first consider that each pair of windings located on the same column can be represented using the equivalent circuit of Fig. 14. Indeed, columns $r_{1}$ and $r_{2}, s_{1}$ and $s_{2}$, and $t_{1}$ and $t_{2}$ are proportional with a factor equal to 2.65. In this way, it remains to find a correct representation of the three primaries. Notice that, in the final circuit, the leakage inductance of Fig. 14 has been entirely located on the righthand side and even moved to the coupler secondary.

3) Equivalent Circuit Diagram for the Three Primaries: Taking advantage of $r_{1}$ and $t_{1}$ winding identities, the three primary transformers we are now studying are decomposed

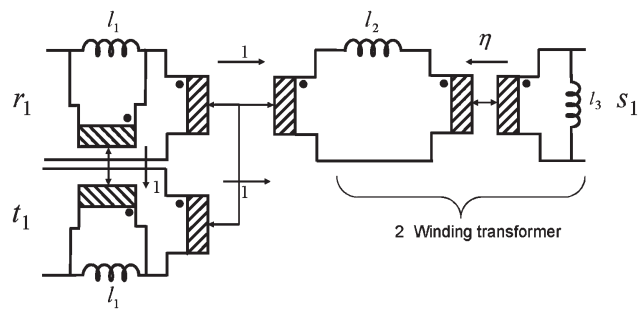

Fig. 15. Initial circuit for the representation of the three primary windings.

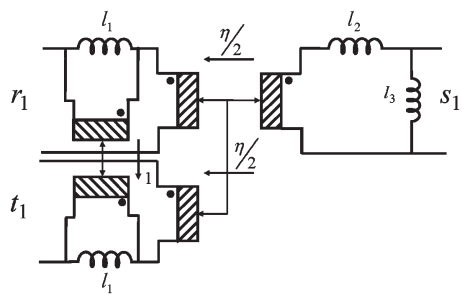

Fig. 16. Final schematic for the three primaries.

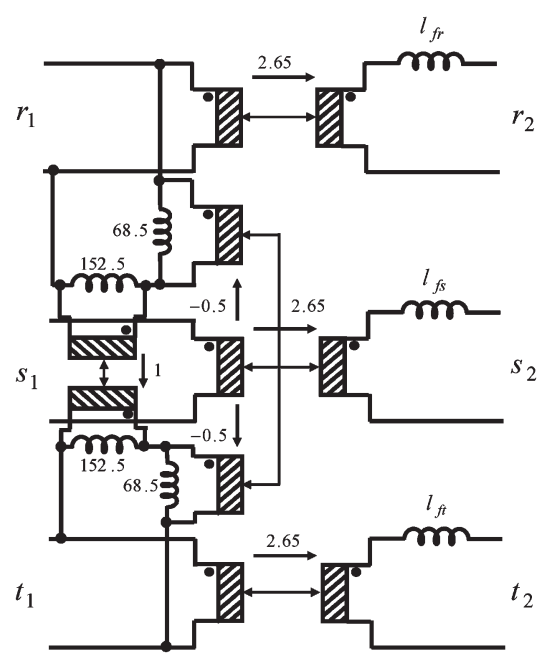

Fig. 17. $2 \times 3$-winding transformer equivalent schematic. Inductance values are in millihenrys.

(Fig. 15) into a two-winding transformer and a winding splitter identical to that described by Fig. 7. Notice that inductances $l_{1}$ are in parallel and they have been split only to increase circuit symmetry.

If $l_{2}$ is moved to the right side of the coupler, a coupler can be suppressed (Fig. 16). In this last figure, four parameters are adjustable and that number is equal to the number of independent matrix coefficients. These parameters can be calculated by identification of the measured inductance matrix with the one deduced from the given circuit

$$
\begin{gathered}
{\left[\begin{array}{ccc}
\frac{1}{2} l_{1}+\frac{\eta^{2}}{4} l_{2}+\frac{\eta^{2}}{4} l_{3} & \frac{\eta}{2} l_{3} & -\frac{1}{2} l_{1}+\frac{\eta^{2}}{4} l_{2}+\frac{\eta^{2}}{4} l_{3} \\
\frac{\eta}{2} l_{3} & l_{3} & \frac{\eta}{2} l_{3} \\
-\frac{1}{2} l_{1}+\frac{\eta^{2}}{4} l_{2}+\frac{\eta^{2}}{4} l_{3} & \frac{\eta}{2} l_{3} & \frac{1}{2} l_{1}+\frac{\eta^{2}}{4} l_{2}+\frac{\eta^{2}}{4} l_{3}
\end{array}\right]} \\
=\left[\begin{array}{ccc}
111 & -69 & -42 \\
-69 & 137 & -68 \\
-42 & -68 & 110
\end{array}\right]
\end{gathered}
$$




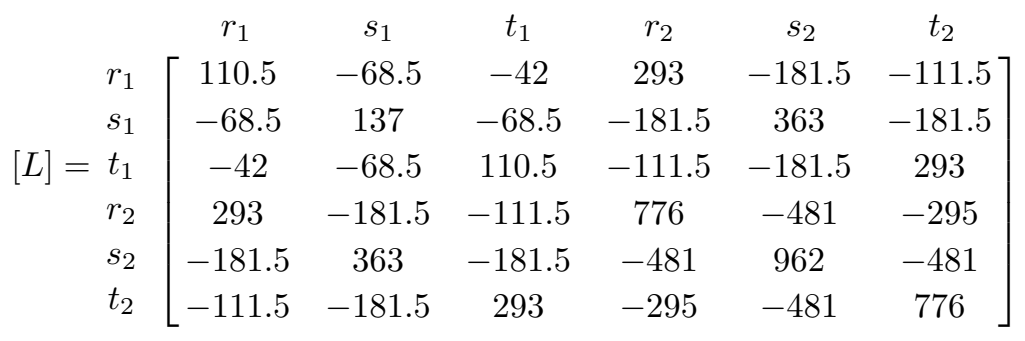

Inaccuracy of measurements and symmetry imperfections explain why some expected equalities are not strictly satisfied. For example,

$$
\begin{aligned}
& L_{r 1 s 1}=L_{s 1 t 1}(-69 \mathrm{mH} \#-68 \mathrm{mH}) \\
& L_{r 1 r 1}=L_{t 1 t 1}(111 \mathrm{mH} \# 110 \mathrm{mH}) .
\end{aligned}
$$

In such a case, average values $(-68.5$ and $110.5 \mathrm{mH}$ ) are taken for calculation. This leads to

$$
\eta=-1 \quad l_{1}=152.5 \mathrm{mH} \quad l_{2}=0 \mathrm{mH} \quad l_{3}=137 \mathrm{mH} .
$$

$l_{2}$ represents the flux generated in central column which is not recovered in another. Its value is very small $(68 \mathrm{mH}+$ $42 \mathrm{mH}-110 \mathrm{mH} \# 0$ ) in comparison to other inductances. This negligible value cannot be accessed through the measured matrix element $[L]$ because of experimental uncertainty. In practice, it is evaluated, as other leakage inductances, by a short-circuit test.

4) Transformer Equivalent Circuit Diagram: Now, we can conclude. To reintroduce secondary windings, we account for the strong coupling which links primary and secondary of a same column, by adding three couplers and three weak leakage inductances (Fig. 17). Because the coupling coefficients involved are very close to 1 , coupling ratio $\eta$ is chosen equal to the transformation ratio 2.65 .

Inductances $l_{\mathrm{fr}}, l_{\mathrm{fs}}$, and $l_{\mathrm{ft}}$ which represent each column leakage, have low values compared with $L_{i j}$ so we can represent them at primary or at secondary indifferently.

The final circuit inductance matrix is deduced from the one established for the three primaries owing to following relations:

$$
L_{I_{2} J_{2}}=2.65^{2} L_{I_{1} J_{1}} \quad L_{I_{1} J_{2}}=2.65 L_{I_{1} J_{1}} .
$$

Short-circuit measurements [8] show that leakage inductances are close to $0.4 \mathrm{mH}$. The resulting matrix, shown at the top of the page, is in good agreement with that measured. This proves that the final equivalent circuit (Fig. 17) is a good representation of the measured inductance matrix.

\section{Measurement Strategy}

In Section III-E, we proposed identifying parameters through a set of impedance and voltage gain measurements. Despite the fact that this is theoretically correct, in practice, impedance measurements are more reliable when a good accuracy is needed. Indeed, modern impedance analyzers offer several efficient compensations for unavoidable wiring. These measuring apparatuses work in a wide range of frequencies and in a wide range of impedances, too. Of course, this advice principally applies to small (less than $1 \mathrm{~L}$ ) components for which these analyzers are usable.

For a two-winding transformer, it is recommended to measure open- and short-circuit impedances seen from both windings. These four impedances are linked, but, if the less accurately acquired one is dropped, the remaining ones allow accurate determination of both leakage inductances and inductance matrix elements.

The previous method applies for a two-winding transformer. However, assuming impedances acquired while zero to $n-1$ inputs are short circuited are measured, it can be extended to an $n$-winding transformer. This is shown in [9] and, in the same paper, practical precautions regarding contact resistances and compensation procedures are addressed as well as accounting for different kinds of losses during inductance and coupling ratio determination.

\section{CONCLUSION}

This paper has presented a method, based on energy considerations, that leads to the equivalent circuit of a lossless transformer, whatever its number of windings. Although the negative inductance concept is cleared up, the employed method only introduces a positive one. To simplify the design, two properties are used: identity of two windings and dominant coupling. All these concepts are illustrated by the elaboration of the equivalent circuits of a three-phase inductor and a six-winding six-column transformer. Resulting circuit of the transformer is compared to measured values. All equivalent circuits given here are usable with any electronic simulation software.

More generally, each time that wires are magnetically coupled and lumped element representation works, methods presented here can be applied. They have been used successfully in some cables and wiring studies.

\section{REFERENCES}

[1] A. M. Urling, V. A. Niemela, G. R. Skutt, and T. G. Wilson, "Characterizing high frequency effects in transformer windings: A guide to several significant articles," in Proc. IEEE APEC, 1989, pp. 373-385.

[2] J.-P. Keradec, B. Cogitore, E. Laveuve, and M. Bensoam, "Des schémas équivalents pour les circuits couplés multi-enroulements," J. Phys. III, vol. 4, no. 4, pp. 751-773, Apr. 1994.

[3] A. Schellmanns, J.-P. Keradec, J.-L. Schanen, and K. Berrouche, "Representing electrical behavior of transformers by lumped element circuits: A global physical approach," in Proc. IEEE IAS Annu. Meeting, Phoenix, AZ, Oct. 3-7, 1999, vol. 3, pp. 2100-2107. 
[4] R. W. Erickson and D. Maksimovic, "A multiple-winding magnetics model having directly measurable parameters," in Proc. IEEE PESC, 1998, pp. $1472-1478$.

[5] S. Cuk and R. D. Middlebrook, Advances in Switched Mode Power Conversion, vol. 1. Pasadena, CA: TESLAco, 1981, pp. 205-218.

[6] A. A. Dauhajre, "Modelling and leakage estimation of leakage phenomena in magnetic circuits," Ph.D. dissertation, Div. Eng. Appl. Sci., California Inst. Technol., Pasadena, Apr. 1986.

[7] S. El-Hamasy and E. I. Chang, "Magnetic modelling for computer aided design of power electronic circuits," in Proc. IEEE PESC, 1989, pp. 635-645.

[8] V. Leitloff, La Modélisation des Transformateurs dans CIRCUIT, $2^{\text {ème }}$ Studienarbeit ed. Grenoble, France: Laboratoire d'Electrotechnique de Grenoble, Jun. 1990, pp. 53-59.

[9] X. Margueron and J.-P. Keradec, "Identifying the magnetic part of the equivalent circuit of a $n$-winding transformer," in Proc. IMTC, Ottawa, ON, Canada, May 17-19, 2005, pp. 1064-1069.

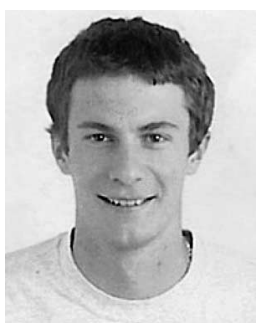

Xavier Margueron was born in Chambéry, France, in 1980. He received the Diplôme d'Ingénieur degree in electrical engineering from the Ecole National Supérieur d'Ingénieur Electricien de Grenoble, France, and the Diplôme d'Études Approfonfies degree from Institut National Polytechnique de Grenoble (INPG), France, both in 2003. He is currently working toward the Ph.D. degree at the Laboratoire d'Electrotechnique de Grenoble, SaintMartin-d'Heres, France.

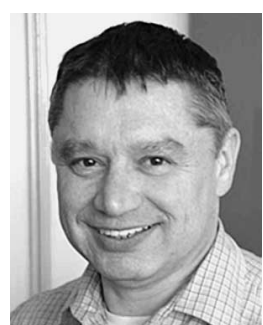

Jean-Pierre Keradec (M'92) was born in Paris, France, in 1947. He received the Doctorat de Physique du Solide degree from the University of Grenoble, Grenoble, France, in 1973.

$\mathrm{He}$ currently teaches courses in analog electronics, signal processing, and measurement systems design at the Institut Universitaire de Technologie of Grenoble and the INPG. He joined the Laboratoire d'Electrotechnique de Grenoble, Saint-Martind'Heres, France, in 1985. His research interests include high-frequency power transformer modeling and measurements related to high-frequency static power converters. 